\title{
Automated Rendezvous and Docking Sensor Testing at the Flight Robotics Laboratory
}

\author{
J. Mitchell/JSC, A. Johnston/MSFC, R. Howard/MSFC, M. Williamson/MSFC, \\ L. Brewster/MSFC, D. Strack/Odyssey Space Research, S. Cryan/JSC
}

\begin{abstract}
The Exploration Systems Architecture defines missions that require rendezvous, proximity operations, and docking (RPOD) of two spacecraft both in Low Earth Orbit (LEO) and in Low Lunar Orbit (LLO). Uncrewed spacecraft must perform automated and/or autonomous rendezvous, proximity operations and docking operations (commonly known as Automated Rendezvous and Docking, AR\&D). The crewed versions may also perform AR\&D, possibly with a different level of automation and/or autonomy, and must also provide the crew with relative navigation information for manual piloting. The capabilities of the RPOD sensors are critical to the success of the Exploration Program.
\end{abstract}

NASA has the responsibility to determine whether the Crew Exploration Vehicle (CEV) contractor-proposed relative navigation sensor suite will meet the $\mathrm{CEV}$ requirements. The relatively low technology readiness of relative navigation sensors for AR\&D has been carried as one of the CEV Projects top risks. The AR\&D Sensor Technology Project seeks to reduce this risk by increasing technology maturation of selected relative navigation sensor technologies through testing and simulation, and to allow the CEV Project to assess the relative navigation sensors.

The first year of this project was focused on a series of "pathfinder" testing tasks to develop the test plans, test facility requirements, trajectories, math model architecture, simulation platform, and processes. The second year of the project will use the information and data collected from the "pathfinder" testing to evaluate the Contractor-proposed sensors. Four highly applicable candidate sensor were identified for the "pathfinder" activities: the Johnson Space Center's (JSCs) Automatic Targeting and Reflective Alignment Concept (AutoTRAC) Computer Vision System (ACVS), which is a camera-based system that uses reflectors on the target vehicle; JSCs Natural Feature Image Recognition (NFIR), which is a camera-based system that does not require reflectors; Marshall Space Flight Center's (MSFCs) Advanced Video Guidance Sensor (AVGS), which is a laser-based system that uses reflectors on the target vehicle; and the Optech Lidar, which is a laser-based system that produces range and intensity data, provided by the Jet Propulsion Laboratory (JPL) for this task.

Sensor characterization and testing for each of these four sensors was conducted at the MSFC Flight Robotics Laboratory (FRL) using the FRL 6-DOF gantry system, called the Dynamic Overhead Target Simulator (DOTS).
The target vehicle for "docking" in the laboratory was a mockup that was representative of the proposed CEV docking systems, with added retroreflectors for the pathfinder sensors and a standoff cross target for visual recognition by the NFIR sensor.

The sensors were tested using four categories of open-loop test trajectories: (1) sensor characterization trajectories designed to test a wide range of performance parameters, (2) CEV-specific trajectories designed to test performance during CEV-like approach and departure profiles, (3) lighting tests designed to evaluate sensor performance under a range of lighting conditions, and (4) failure and extreme conditions tests designed for evaluating sensor performance under more extreme conditions as might be induced during a spacecraft failure or during contingency situations. This paper describes the test development, test facility, test preparations, test execution, and test results.

\subsection{INTRODUCTION}

AR\&D for the CEV requires a suite of relative navigation sensors that meet performance requirements and have operational characteristics and failure modes that are well understood. NASA has the responsibility to approve (or disapprove) the Contractor-proposed sensor suite. In order to do this, NASA will need to perform independent testing as early as possible in order to minimize cost and schedule impacts.

The Exploration Systems Technology Development (ETDP) AR\&D Sensor Technology Project has three major activities: the development of a relative navigation sensor database, relative navigation sensor testing, and relative navigation sensor modeling and simulation. The sensor database is used to capture sensor information and data. The sensor testing task allows NASA to obtain hands-on experience with the operational and performance characteristics of the sensors. The sensor modeling and simulation task provides a means by which to create and validate sensor models to be used in the design and development of the guidance, navigation, and control (GN\&C) system. For the fiscal year (FY) 2006 (FY06), the project will apply these activities to a set of "pathfinder" sensors that will be used to prepare for evaluation of the CEV Contractor-proposed sensors in FY07.

The AR\&D Sensor Technology Project has three primary objectives related to the test planning and execution: 
1. To develop a comprehensive test plan for testing relative navigation sensors that the CEV Project can use as the basis for independent assessment and testing of $\mathrm{CEV}$ contractor-proposed relative navigation sensors.

2. To execute the test plan to evaluate the selected relative navigation sensor technologies, as defined in this project, in order to understand the performance and operational characteristics.

3. To use test results to develop a representative math model for each of the selected relative navigation sensors.

\subsection{TEST ARTICLES}

This section describes the details of the four test articles. For FY06 testing, four test articles have been selected as pathfinders to pave the way for testing CEV contractorproposed sensors (or functional equivalents) in FY07. The team surveyed the sensors used in the Defense Advanced Research Project Agency's Orbital Express (a satellite servicing and resupply technologies program), Lockheed Martin's Hubble Recovery Vehicle design, the United States Air Force Research Laboratory's XSS-11 satellite system, and the CEV reference design. The goal was to choose pathfinder sensors with the following attributes:

1. They are representative of the types of sensors likely to be included in the $\mathrm{CEV}$ contractor-proposed relative navigation sensor suite.

2. Hardware and software domain expertise is resident within the team.

3. Hardware and software is available with minimal acquisition cost to this project.

4. The operational range is within the FRL dimensions for final approach and docking.

Four highly applicable candidate sensor were identified for the "pathfinder" activities: the Johnson Space Center's (JSCs) Automatic Targeting and Reflective Alignment Concept (AutoTRAC) Computer Vision System (ACVS), which is a camera-based system that uses reflectors on the target vehicle; JSCs Natural Feature Image Recognition (NFIR), which is a camera-based system that does not require reflectors; Marshall Space Flight Center's (MSFCs) Advanced Video Guidance Sensor (AVGS), which is a laser-based system that uses reflectors on the target vehicle; and the Optech Lidar, which is a laser-based system that produces range and intensity data, provided by the Jet Propulsion Laboratory (JPL) for this task.

\section{AutoTRAC Computer Vision System (ACVS)}

The ACVS is a camera-based system that employs the use of light emitting diodes (LEDs) and specific targets composed of either mirrors or reflective surfaces to determine a relative state (range, azimuth, elevation, and roll, pitch, and yaw). ACVS elements are shown in Figure 1.

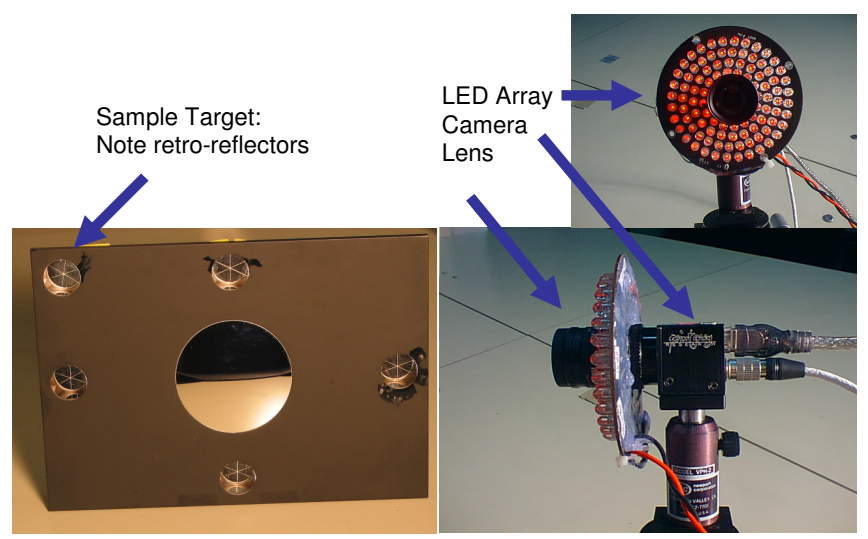

Figure 1. AutoTRAC Computer Vision System (ACVS)

The ACVS is composed of a charge-coupled device camera with LED array using a specific target. The operating principle is a 6-DOF pose based on a known target pattern. The first video frame is taken with LEDs on; the second video frame with LEDs off. The two frames are then subtracted from each other, eliminating the background while leaving the bright return from the retros. Standard image segmentation techniques are then used to determine the retros' locations in the image. The correspondence between the potential target features (retro blobs) and the known target model is then determined. Finally, a nonlinear least-squares fit of the image data is done to determine the 6-DOF pose of the target object with respect to the camera.

The ACVS was used for the Dexterous End Effector Detailed Test Objective (DTO) for STS-62, DTOs for STS85 and STS-95, and the AERCam navigation sensor for docking (air-bearing table).

The ACVS capabilities vary based on the focal length of the camera lens selected and on the size of the ACVS target. Multiple ACVS targets are supported which would allow for reasonable pose results at long range using a large target, while using a smaller target at close range. The parameters shown in Table 1 are based on those used for the FRL PMA mockup tests - a single fixed 9mm lens, 1024x768 imager, and 1 long and 1 short range ACVS target: 
Table 1. ACVS Operational and Performance Parameters

\begin{tabular}{|c|c|}
\hline FOV & $\begin{array}{l}28 \text { degrees horizontal x } 21 \text { degrees vertical - } \\
9 \mathrm{~mm} \text { lens }\end{array}$ \\
\hline Range & $\begin{array}{l}0.75-40 \text { meters. } \\
\text { The range for a short range target is } \\
\text { approximately } 0.75-20 \text { meters and a long } \\
\text { range target (LRT) is approximately } 8-40 \\
\text { meters. }\end{array}$ \\
\hline Accuracy & $\begin{array}{l}\text { Predicted accuracies of } 0.001 \text { inch and } 0.1 \\
\text { degree, operational expectation is } 1 \text { percent } \\
\text { of range and } 1 \text { degree in pitch, yaw, and roll. }\end{array}$ \\
\hline Update rate & $15 \mathrm{~Hz} @ 30 \mathrm{hz}$ frame rate \\
\hline $\begin{array}{l}\text { Target } \\
\text { angles }\end{array}$ & LRT/SRT up to \pm 10 degrees \\
\hline $\begin{array}{l}\text { Power } \\
\text { consumption }\end{array}$ & 7.2 watts@18 VDC \\
\hline Mass & $\begin{array}{l}0.45 \text { kilograms each }- \text { sensor/LED and } \\
\text { electronics box }\end{array}$ \\
\hline Dimensions & $\begin{array}{l}12 \times 10 \times 10 \text { centimeters sensor, } 13 \times 13 \times 6 \\
\text { centimeters electronics }\end{array}$ \\
\hline $\begin{array}{l}\text { Data } \\
\text { interface }\end{array}$ & $\begin{array}{l}\text { IEEE } 1394 \text { from the ACVS camera to the } \\
\text { data processing computer }\end{array}$ \\
\hline
\end{tabular}

Failure modes and FDIR for the ACVS are the following:

- No pose determined due to too many potential target features (blobs/retros) or not enough targets features to match the target model.

- Pixel jitter, partial retro-occlusion, or the retros sitting on the edge of the camera image frame can produce poor pose estimation - right pose, but slightly wrong values due to mis-determination of the true center of the target features.

- Internal software checks for matching retro-design dimensions to the derived optical properties can cause rejection of a target feature (e.g. retro blob not "round").

- Least-squares-fit residuals of the pose must pass a static "goodness" limit before being marked as a valid pose.

- Imaging (brightness) of the target retros can be externally manipulated by manual control of the LED power input ( $10.0 \mathrm{VDC}->18 \mathrm{VDC}$ range)

- The software can adjust the camera exposure at each cycle based on pixel brightness value, to help achieve better retro imaging.

For data acquisition, camera images are sent to a personal computer (PC) where the images are processed in pairs to estimate the target's 6-DOF pose with respect to camera. There is no input requirement and the data output rate is a maximum of $15 \mathrm{~Hz}$ when using the PMA FRL test's $1024 \times 768$ pixel cameras. A $60 \mathrm{~Hz}$ rate is available when using 640x480 pixel cameras.

Data output types include 4 timestamps in several formats (first frame start, second frame end, algorithm start, pose data output), pitch, yaw, roll, $x, y, z$, pose-fit, and then "blobs" data. "Blobs" are the individual retro-returns as seen by the camera, and include size and location in the camera frame. Raw video data can be recorded, but the files will be rather large.

The ACVS hardware and software components used for this test program are based on a 1997 development effort at JSC. The camera is a 2006 Point Grey 1024 x 768 black and white digital camera. The software has been updated during various development efforts over the last several years.

\section{Advanced Video Guidance Sensor (AVGS) Overview}

The AVGS is a system that combines advanced optical and electronic ranging technologies. The AGVS and a target are shown in Figure 2.
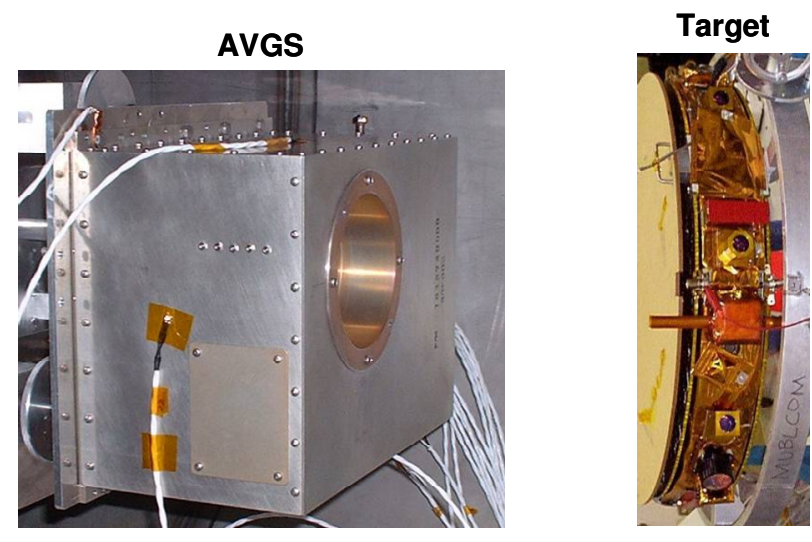

Figure 2. Advanced Video Guidance Sensor (AVGS)

The AVGS is a video-based sensor that uses lasers to illuminate filtered retro-reflective targets. The operating principle is that the first picture is taken while the foreground laser illuminates the target, and the second picture taken while the background laser illuminates the target. The second picture is subtracted from the first picture and the remaining spots are matched to the target and used to compute relative position and attitude.

The AVGS is an advanced version of the Video Guidance Sensor (VGS) developed by NASA MSFC. The VGS flew on STS-87 and STS-95. The AVGS flew on the Demonstration of Autonomous Rendezvous Technology (DART) spacecraft in 2005 and will fly on the Orbital Express in November 2006. 
The AVGS operational and performance parameters are shown in Table 2.

Table 2. AVGS Operational and Performance Parameters

\begin{tabular}{|l|l|}
\hline FOV & 16 x 16 degrees \\
\hline Range & $\begin{array}{l}0.75-300 \\
\text { The range for a short-range } \\
\text { target (SRT) is 0.75-20 meters } \\
\text { and a long range target (LRT) is } \\
10-300 \text { meters. }\end{array}$ \\
\hline Accuracy (at dock) & \pm 13 millimeters, \pm 0.3 degrees \\
\hline Update rate & $5 \mathrm{~Hz}$ and $25 \mathrm{~Hz}$ \\
\hline Target angles & $\begin{array}{l}\text { LRT up to } \pm 27 \quad \text { degrees } \\
\text { SRT up to } \pm 12 \text { degrees }\end{array}$ \\
\hline Power consumption & 20 watts \\
\hline Mass & 9.1 kilograms \\
\hline Dimensions & $30.5 \times 25.4 \mathrm{x} \mathrm{17.8} \mathrm{centimeters}$ \\
\hline Data interface & $\begin{array}{l}\text { RS-422 between the AVGS and } \\
\text { the data acquisition computer }\end{array}$ \\
\hline
\end{tabular}

Failure modes include the following:

- Sun in the FOV will generally cause a loss of tracking, but the sensor will recover automatically once the FOV is clear.

- If the entire target (either SRT or LRT) is not in the FOV, that target will not be recognized or tracked, but the sensor will keep attempting to acquire the target.

- Single-event-upsets can cause various problems. AVGS uses watchdog timers and other software and hardware error detection and correction methods.

- Failure of thermoelectric coolers can cause laser problems such as wavelength drift or overheating. Temperature sensors monitor various temperatures at 5 $\mathrm{Hz}$.

The AVGS is cabled to ground support equipment (GSE) with a laptop computer running GASCAR [need to define?] software to command the AVGS and record data. The AVGS requires a seed range input of \pm 25 percent of the actual range to acquire the target. The data output rate is 5 $\mathrm{Hz}$ or $25 \mathrm{~Hz}$

Output of processed data is range, azimuth, elevation, roll, pitch, and yaw, and the data is stored in a raw format with health and status, range, azimuth, elevation, angle quaternion, and spot centroid information.

The AVGS test article used for these tests is serial number 2 from the DART program and was used to test the DART AVGS flight software and to support the testing of the Orbital Express AVGS.

\section{Optech LIDAR}

The Optech LIDAR system uses lasers to measure distance and/or speed to reflective surfaces. The Optech LIDAR is shown in Figure 3.

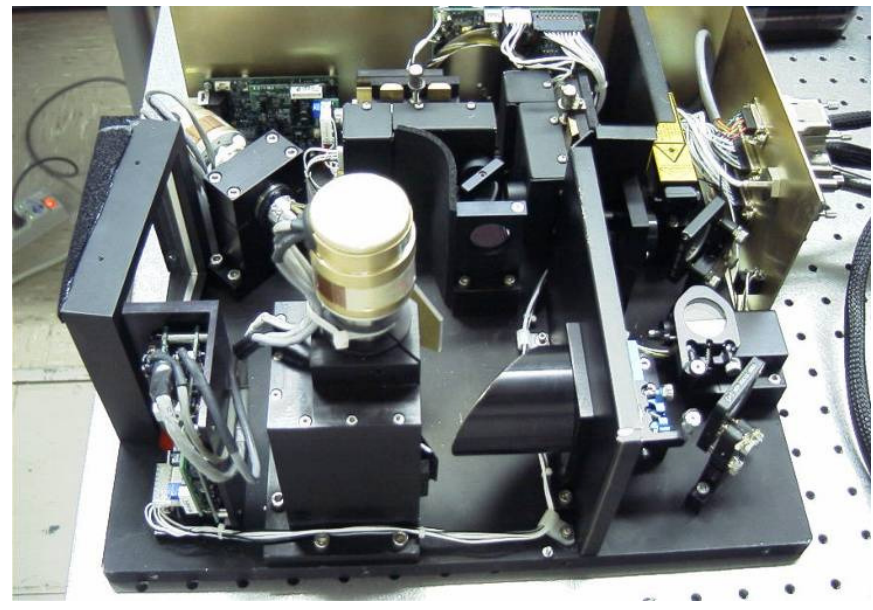

Figure 3. Optech LIDAR

The Optech LIDAR is a pulsed LIDAR system $(10 \mathrm{kHz})$ that is passively Q-switched to approximately 2 microJoules per pulse. The operating principle is time-of-flight with a programmable scanner at $10 \times 10$ degrees.

The Optech LIDAR was part of the United States Air Force Research Laboratory XSS-11 micro-satellite program.

\section{Performance}

The Optech LIDAR features are as shown in Table 3.

Table 3. Optech LIDAR Operational and Performance Parameters

\begin{tabular}{|l|l|}
\hline Field of regard & $10 \times 10$ degree field of regard \\
\hline $\begin{array}{l}\text { Range to } \\
\text { surface }\end{array}$ & $\begin{array}{l}2 \text { kilometer range to surface (albedo }> \\
0.25)\end{array}$ \\
\hline $\begin{array}{l}\text { Range to } \\
\text { retroreflector }\end{array}$ & $5+$ kilometer range to 7millimeter retro \\
\hline Output rate & $\begin{array}{l}10 \mathrm{kHz} \text { samples }-110 \times 10 \text { scan per } \\
\text { second }\end{array}$ \\
\hline Data interface & $\begin{array}{l}\text { RS-422 between the Optech LIDAR and } \\
\text { the data acquisition computer }\end{array}$ \\
\hline
\end{tabular}

Failure modes include the following:

- Scanner failure - Actuator failure (mechanical, electrical, or software) results in ranging still working and limited angular knowledge. 
- Laser failure - Radiation or electrical failure results in loss of device.

- Receiver failure - Thermal or electrical failure results in loss of device.

- Software failure - Results in a fiducial false-positive or improper scanning.

- FDIR is via normal software fault protection.

Data acquisition is accomplished by using a laptop to collect the LIDAR scans, each consisting of up to $10 \mathrm{~K}$ angle, intensity, and range measurements. There are no input requirements and the data output rate is $1 \mathrm{megabit} / \mathrm{second}$.

The Optech LIDAR test article used for these tests is a prototype LR1 unit that was procured in 1998.

\section{Natural Feature Image Recognition (NFIR) Overview}

The NFIR is a model-based state estimation system that uses video images. The operating principle is to search the first image for the bounding box of the target vehicle, locate high-contrast target features, and match the target image features to the 3-D model and compute pose (position and orientation). The Kalman filter is used to estimate state (position, orientation and first-time derivative) from pose.

The NFIR was tested using simulated video of the H-II Transfer Vehicle (HTV) as seen from the exterior ISS camera during terminal approach. It was also tested using simulated video of the Hubble Space Telescope aft bulkhead during terminal approach. The NFIR elements are shown in Figure 4.

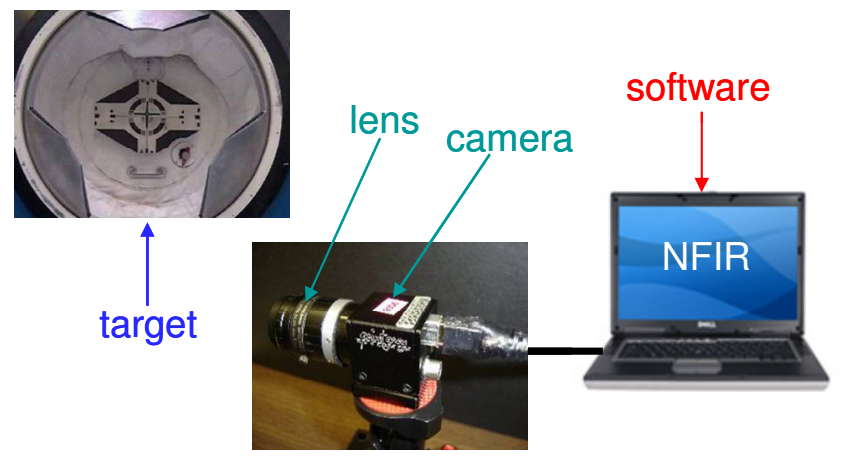

Figure 4. Natural Feature Image Recognition (NFIR)

Pose estimation can use any high-contrast features on the target vehicle for which a 3-D model exists; no special targets are required.

Accuracy from the test using simulated video of the HTV is as defined in Table 4.
Table 4. NFIR Operational and Performance Parameters

\begin{tabular}{|l|l|}
\hline Range accuracy & $\begin{array}{l} \pm 3 \text { percent of range with perfect camera } \\
\text { calibration }\end{array}$ \\
\hline $\begin{array}{l}\text { Range rate } \\
\text { accuracy }\end{array}$ & $\pm 0.06 \mathrm{~m} / \mathrm{sec}$ \\
\hline $\begin{array}{l}\text { Attitude } \\
\text { accuracy }\end{array}$ & \pm 2 degrees \\
\hline $\begin{array}{l}\text { Attitude rate } \\
\text { accuracy }\end{array}$ & $\pm 0.4 \mathrm{deg} / \mathrm{sec}$ \\
\hline Data output rate & $2 \mathrm{~Hz}$ \\
\hline Data interface & $\begin{array}{l}\text { IEEE } 1394 \mathrm{~b} \text { from the NFIR camera to } \\
\text { the data acquisition computer }\end{array}$ \\
\hline $\begin{array}{l}\text { Camera power } \\
\text { (per camera) }\end{array}$ & $\begin{array}{l}12 \mathrm{Vdc}, \text { less than } 2.0 \text { amps via IEEE- } \\
1394\end{array}$ \\
\hline
\end{tabular}

The results in Table 4 above apply from 260 meters to 65 meters with a $48 \mathrm{~mm}$ lens. Test accuracy improves from 65 meters to 10 meters with $9 \mathrm{~mm}$ lens. The NFIR should work at shorter range with a shorter focal length, but it has not been tested inside 10 meters.

Failure modes include the following:

- The camera does not produce a useful image under these conditions:

- Scene brightness exceeds exposure control capability

- Hardware failure

- The image and 3-D model features of the target will be incorrectly matched if:

- The search area prediction was wrong due to an error in the previous pose

- The image motion of the features is larger than the search area

- The computer fails

- The pose estimation fails because of a small number of features due to:

- Poor exposure

- Partial target vehicle occlusion

- Small target vehicle size in the image

For data acquisition and control, the computer receives an image from the camera, computes the pose using image and internal camera calibration, estimates the state, and switches to the other camera when target gets close. The start position 
and orientation of the target in world coordinates are required inputs.

The NFIR camera is a 2006 Point Grey 640 x 480 black and white digital camera. The software has been under development since 2003.

\subsection{TEST FACILITY}

MSFC's Flight Robotics Laboratory (FRL) was developed to provide a single area in which avionics and robotic hardware and software could be tested in a full 6-degree-offreedom (DOF) closed loop simulation. The FRL objective was to provide a full scale, integrated simulation capability for the support of the design, development, integration, validation, and operation of orbital space vehicle systems. The FRL is built on developed technologies such as airbearing floors, servo driven overhead robotic simulators, precision targets, gimbals, 3-DOF mobility units, and manipulator and visual system evaluation facilities.

The FRL consists of two major testing facilities. The flat floor, a $13.41 \times$ 26.21-m (44 x 86-ft) precision air-bearing floor, the largest of its kind, which uses two self-contained mobility units called the Small Air Sled (SAS) and the Large Air Sled (LAS). Both units are capable of 3-DOF motion. and can be used to simulate docking between two separate spacecraft. An 8-DOF overhead gantry, called the Dynamic Overhead Target Simulator (DOTS), provides a 500-lb payload capability for simulating relative motion with respect to a fixed target on the facility floor, shown in Figure 5. A computer system provides inverse kinematics and allows the gantry to act as a target or as the 6-DOF rendezvous vehicle. A "jog" panel, located near the computer terminal, is used to switch the DOTS system into Manual Mode, Computer Mode, or Idle Mode. A DOTS panic button is located on the jog panel and main cutoff switches are located at strategic locations in the floor area. The DOTS has 8 "joints" or DOF - bridge, trolley, waist, shoulder, extension, roll, yaw, and pitch. DOTS uses SICK DME 3000 rangefinders with an accuracy of $+/-5 \mathrm{~mm}$ and Stegmann Coretech CA25 rotary encoders with a repeatability of 0.005 degrees. The resolution at the end of the arm (cumulative through all the joints) is a translational combined error $=0.03$ inch and a rotational combined error $=.022$ degrees.

The test team evaluated the SAS and the DOTS systems and decided to use DOTS for open-loop testing in order to provide sensor characterization in the 6-DOF regime. An evaluation was made as to whether to mount the sensor or the target mockup on the DOTS gantry. There are three main reasons for mounting the target mockup on the moving DOTS platform and keeping the sensors on a fixed test stand:
- The Optech LIDAR is not eyesafe, so safety for lab personnel can be better controlled if the LIDAR is in a fixed position.

- The Optech LIDAR in particular will be most sensitive to the vibration and flex of the moving DOTS platform, but other sensors will also be affected.

- It will be simpler to provide power and data interfaces to the sensors if they are on a fixed test stand instead of on DOTS.

There are drawbacks to this configuration-there may be some test conditions under which the DOTS motion would not be able to replicate the dynamic angular rate of the target vehicle.

The FRL also has a solar simulator that is mounted to a 2DOF carriage with six 6-kVA lights. It operates independently of DOTS. Lighting is variable along the north track in an east-west direction and light pointing is available.

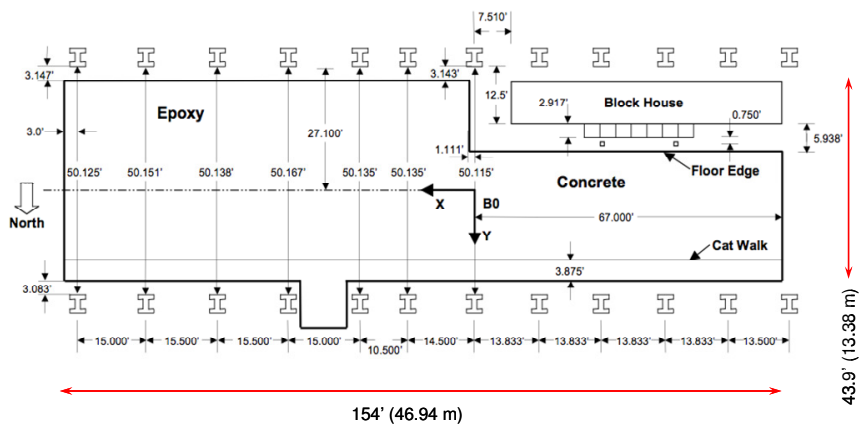

Figure 5. FRL Floor Plan

\section{Truth Data}

Truth data for the FRL trajectories are obtained with the use of a Leica Laser Tracker LTD800 with T-Probe/T-Cam accessories. Range and accuracy are as defined in Table 5.

Table 5. Leica Laser Tracker Range and Accuracy

\begin{tabular}{|l|l|}
\hline 3-DOF (LTD800 stand-alone) \\
\hline Maximum range & 40 meters \\
\hline Measurement accuracy & 0.001 inch \\
\hline 6-DOF (LTD800 with the T-Probe) \\
\hline Maximum range & 15 meters \\
\hline Measurement accuracy & $\begin{array}{l}15 \text { micrometers }+6 \\
\text { micrometers/meter }\end{array}$ \\
\hline
\end{tabular}

The T-Probe is a small device that communicates with the laser tracker. The tracker collects the position (including orientation) of the T-Probe during testing. 
The T-Cam is a camera system that is located on top of the laser tracker. It tracks the T-Probe and determines its orientation through commands of light-emitting diodes on the body of the T-Probe. This sensor has a 15-meter range when used in a 6-DOF mode of operation.

Also, during the 6-DOF mode of operation, the angular limitations are \pm 45 degrees of elevation (with respect to the tracker head) and \pm 45 degrees on the T-Probe (with respect to the T-Probe $\mathrm{z}$-axis).

Prior to testing, the laser tracker is configured to record data in the facility fixed frame B0. The sensor reference frame (the stationary frame under test) must be known with respect to the facility B0 frame so that the relationship of the laser tracker position within that frame can be determined. The data collected will be the T-Probe position and orientation in the B0 reference frame. The relationship of the target frame (D1 DOTS reference frame) to the T-Probe frame will be determined and used in post-processing to estimate the target frame position with respect to the sensor frame. Figure 7 shows the T-Probe reference frame.

Currently, the Leica stores data at a rate of 5 megabytes/hour when using a $25-\mathrm{Hz}$ sample.

\section{Test Facility Limitations}

The Leica laser truth system is 6-DOF for 15 meters. This requires that the trajectories be split up within 15-meter segments so that the truth sensor can be repositioned for increasing ranges.

The DOTS encoder can provide truth data at a resolution of 0.1 inch and 0.01 degrees.

The blockhouse in the southwest corner may interfere with some trajectories. Pretest checkout of trajectories must be done to determine bad flight patterns. The checkouts will be accomplished with a desktop model of the safety and kinematic routines used by the real-time code.

The FRL DOTS limits the azimuth and elevation angles.

The maximum movement of the gantry $(0.3 \mathrm{~m} / \mathrm{sec})$ is restricted to the following:

- $\quad$ Maximum radial distance: 38 meters

- Maximum lateral distance: 13.3 meters

- $\quad$ Maximum up/down distance: 4.6 meters

The maximum rotation of the gantry $(1 \mathrm{deg} / \mathrm{sec})$ is restricted to the following rotations:

- Pitch: $\quad \pm 28$ degrees
- Yaw: $\quad \pm 30$ degrees

- Roll: 360 degrees

\subsection{TARGET VEHICLE DOCKING MOCKUP}

The design of the target vehicle docking mockup is an important component of this test program. Two docking systems are under consideration for the CEV: the Androgynous Peripheral Attachment System (APAS) and the Low Impact Docking System (LIDS). LIDS has been selected as the docking system for the CEV docking to the Lunar Surface Ascent Module (LSAM). As of the initiation of this test program, the Constellation Program has not decided whether the CEV will use APAS or LIDS when docking to the International Space Station. Because of the ambiguity, the project team decided to use a simple docking system representation that could be applicable to either docking system.

For the three pathfinder sensors that will rely on retroreflectors (ACVS, AVGS and the Optech Lidar), the shape and details of the docking system mockup are not important as long as the retroreflector placement is in a reasonably realistic geometric pattern. However, the NFIR system uses natural features of the target object. In order to create a simple but relevant target feature set, it was decided that the standoff cross target used for Shuttle docking to the ISS would be a good choice for short range and a docking ring the same size and shape of the APAS mechanism would be a good choice for long range. In addition, the NFIR software already included a model of the standoff cross target, so minimal software development would be required. The target mockup requirements for the pathfinder sensors are described in Table 6.

Table 6. Target Mockup Requirements for Pathfinder Sensors

\begin{tabular}{|c|}
\hline NFIR \\
\hline $\begin{array}{l}\text { Existing software can track the ISS docking target } \\
\text { (nonflight spare) with triangular mounting plate. }\end{array}$ \\
\hline $\begin{array}{l}\text { The target plate needs to be mounted in the center of the } \\
\text { docking tunnel. }\end{array}$ \\
\hline $\begin{array}{l}\text { The target plate will be located one foot inside the } \\
\text { docking tunnel so that the top of the stand-off cross is in } \\
\text { the same plane as the APAS docking pressure plate. }\end{array}$ \\
\hline AVGS long-range (LR) and short-range (SR) targets \\
\hline $\begin{array}{l}\text { The AVGS uses a pattern of three targets (DART } \\
\text { pattern) for LR and a small target for SR. }\end{array}$ \\
\hline ACVS targets \\
\hline The ACVS uses five retros for the target pattern. \\
\hline $\begin{array}{l}\text { For this configuration, use the existing three AVGS LR } \\
\text { targets and add two more AVGS LR retros (laboratory } \\
\text { test confirmed that ACVS could track AVGS targets) } \\
\text { and one SR ACVS target pattern. }\end{array}$ \\
\hline Optech LIDAR \\
\hline
\end{tabular}


Will use the AVGS/ACVS target pattern defined above.

Figure 6 shows the sensor reflector/target configuration and Figure 7 shows the target vehicle docking mockup design.
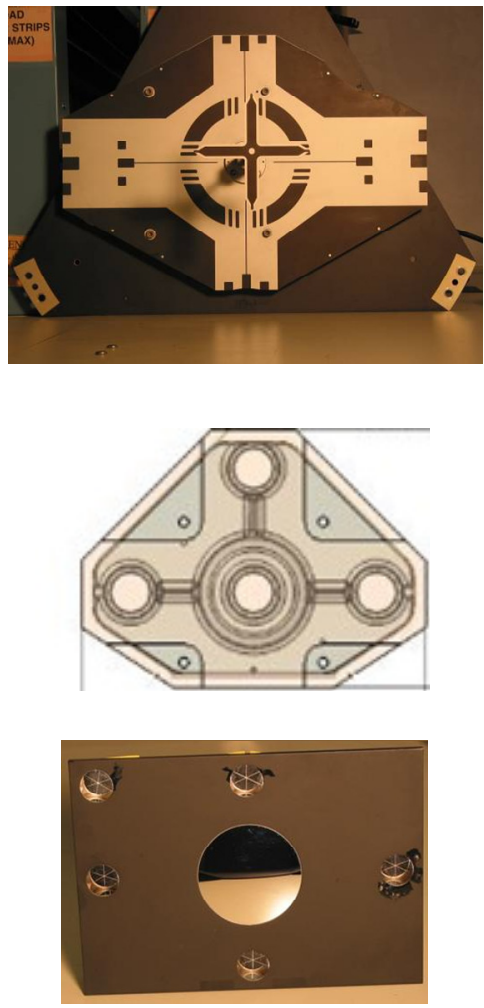

Figure 6. Sensor Reflector/Target Configuration

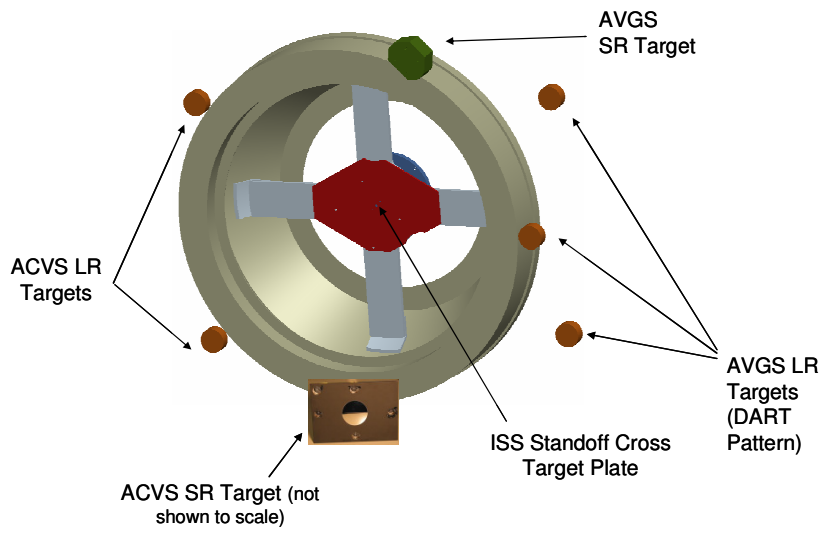

Figure 7. Target Vehicle Docking Mockup Design

The resulting target mockup represents the general size and shape of the ISS PMA-2 tunnel and the APAS mechanism. Details such as hooks, wiring, and petals are not modeled. The mockup configuration was selected to be within the mounting requirements of the FRL DOTS 6-DOF gantry, meeting weight and clearance constraints. The reflector targets are placed around the perimeter of the mockup to minimize design changes to the sensors that use them (locations were not based on real CEV vehicle constraints). Figure 8 shows the target vehicle docking mockup as shown in the JSC Building 9 hi-bay. The white docking ring as shown was removed and integrated with the standoff cross target and the retroreflectors as shown in Figure 9.

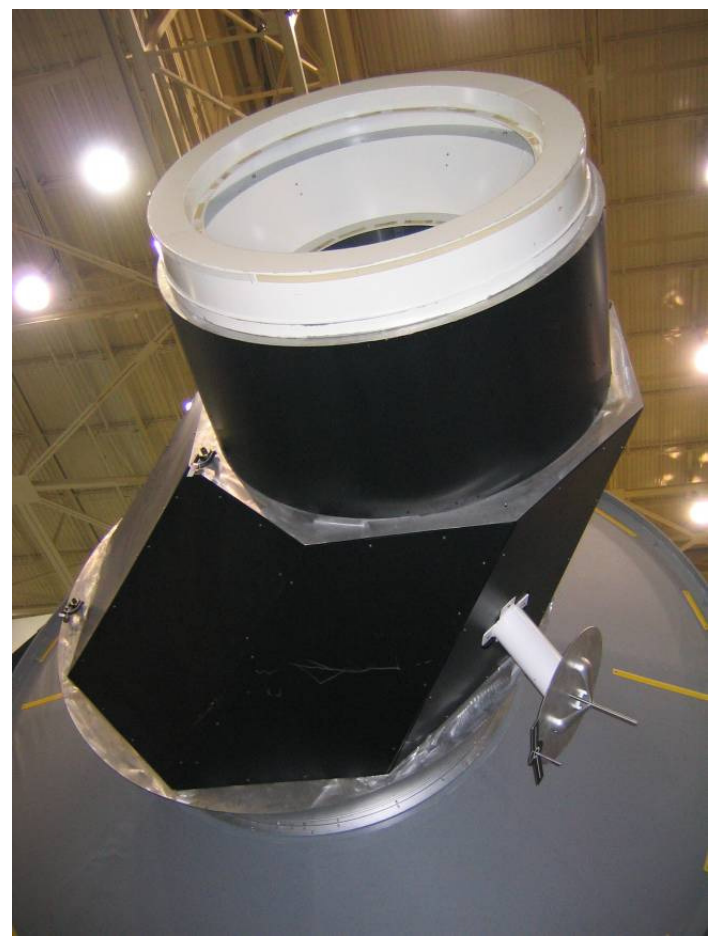

Figure 8. Target Vehicle Docking Mockup Mounted in the JSC Building 9 Hi-bay

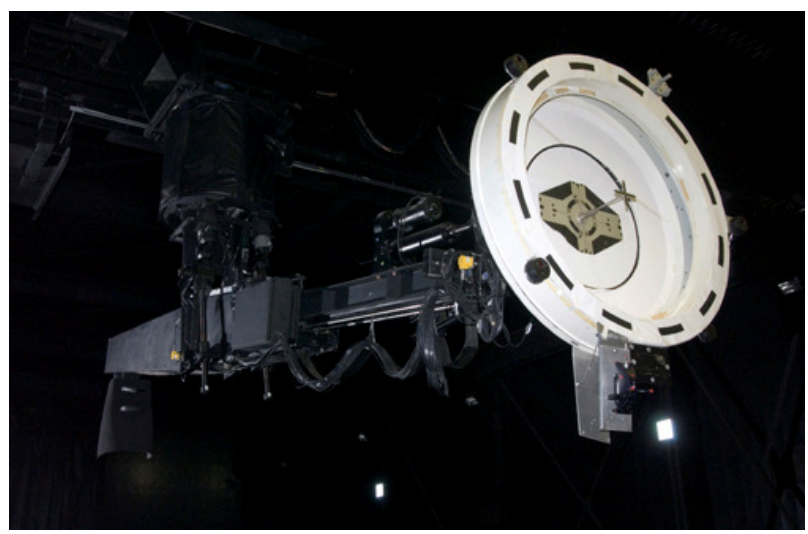

Figure 9. Target Vehicle Docking Mockup Mounted on the FRL DOTS

\subsection{TEST SETUP}

The test sensor was first mounted to a test stand within the FRL as shown in Figure 10. The actual position was surveyed using a coordinate measuring machine (CMM). This CMM is a Leica LTD 800 and is used as a 'truth measurement' for evaluating the sensor performance. The 
LTD 800 is a $40-\mathrm{m}$ laser tracker that uses an interferometer and absolute distance meter (ADM) to measure position of objects in space to within $0.1 \mathrm{~mm}$. The Leica also uses a Tprobe and a camera for 6 DOF measurements. The T-probe is a small active targeting device that provides attitude information within 15 meters. The camera is used to record LED's on the T-probe for positioning information. The Tprobe is limited to about +/- 45 degrees during the 6DOF mode of operation. The Leica LTD 800, combined with the 6-DOF T-probe, provides relative range, bearing, and orientation.

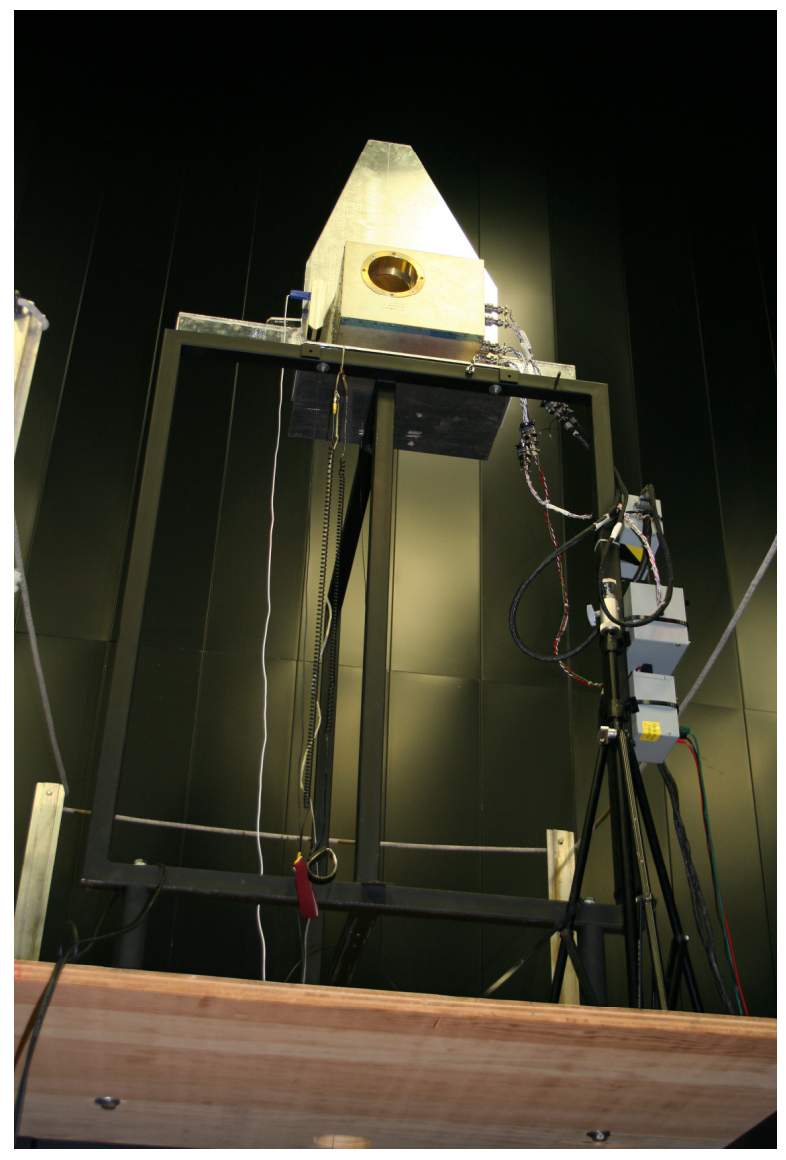

Figure 10. AVGS Mounted on Test Stand

The sensor target was mounted onto the mockup on the DOTS as shown in Figure 11. The relationship, or vector, from the T-probe to the sensor target is developed, again with the Leica. Lastly, the Leica is surveyed into the facility (the B0 frame) using know check points. So before any testing is started, the relationships between probe to sensor target, facility to sensor, and facility to Leica are established. The DOTS server provides a timestamp to the sensor controller and generates a TTL level pulse to trigger the Leica to record relative position and orientation for later comparison with sensor output.

Figure 12 develops a simplified model of the testing. Since the Leica and test sensor don't use the same target, the truth data must be transformed into a common frame for comparison. By simple vector addition,

$$
\overline{R_{\text {SENSOR }}}=\overline{R_{\text {LEICA POSITION }}}+\overline{R_{\text {LEICA TARGET }}}+\overline{R_{\text {TARGETS }}}
$$
Eq. 1

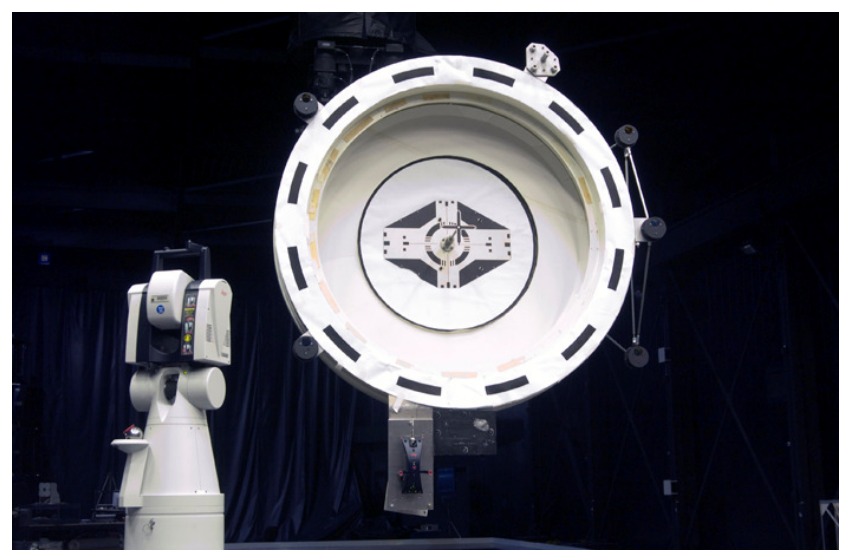

Figure 11. Leica CMM in foreground with DOTS mockup and Leica target mounted underneath

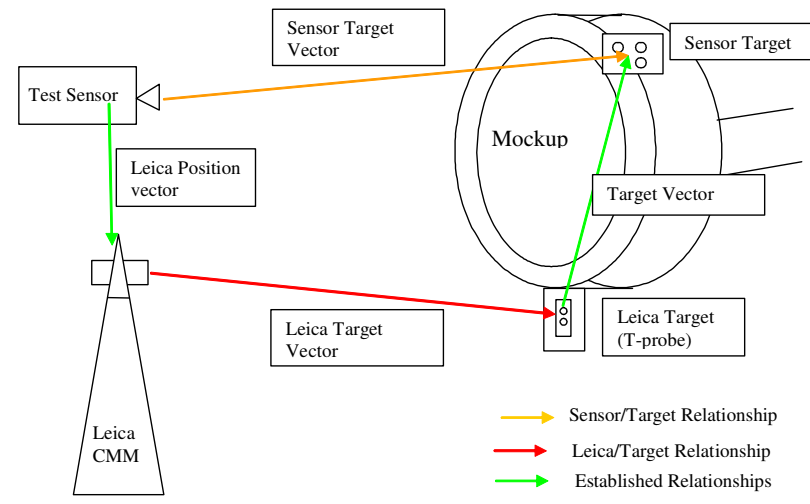

Figure 12. Model of Leica/Sensor Vectors

The addition of the Leica position vector (measured real time during testing) to the Leica measurement of the sensor target from the t-probe location (fixed vector relationship) and the sensor to Leica recording frame (also fixed) is equivalent to the sensor to sensor target vector (Eq. 1). During the test, the sensor records target position and the Leica records its own T-probe position. After the test, the data is post-processed with direction cosine transformation matrices to allow comparison of the Leica data to the test sensor coordinate frame. However, this simple model must be modified to allow for the robot arm movement.

\section{DOTS Vectors}

The DOTS uses its own encoders to 'fly' a closed loop trajectory. For these tests, the end of the mockup was defined to be coordinate frame D1. A second frame (near 
the sensor position) was defined to be D2, which is simply a frame in space and not on any physical feature. D1 and D2 are defined so that the robot can move consistently and give a repeatable trajectory. The DOTS input file defining the trajectory is in the D1 to D2 frame. If the D2 frame was placed on the sensor, it would move from test to test because the each test sensor position within the facility is different. The actual end of robot arm (yet another coordinate frame B8) to the mockup frame (D1) was determined by the Leica (note: DOTS records the encoders B8 tip motion in the B0 frame along with the B8 to B0 commanded tip position.) The Leica is placed in the facility frame (B0), so that its measurements are essentially B0 to D1 (which is the movement of the mockup within the facility) (See Figure 4).

\section{Final Analysis}

The DOTS encoders give us a position and attitude as the robot moves- a B8 to D2 relationship. This is transformed to the D1 to D2 frame. The Leica gives us the true measurement, a Leica (B0) to T-Probe relationship. This is also transformed to the D1D2 framework as shown in Figure 13. The sensor to target frame (not shown) is also transformed into the D1D2 framework. So we now have 3 trajectories, sensor, Leica, and DOTS, which can be compared to one another.

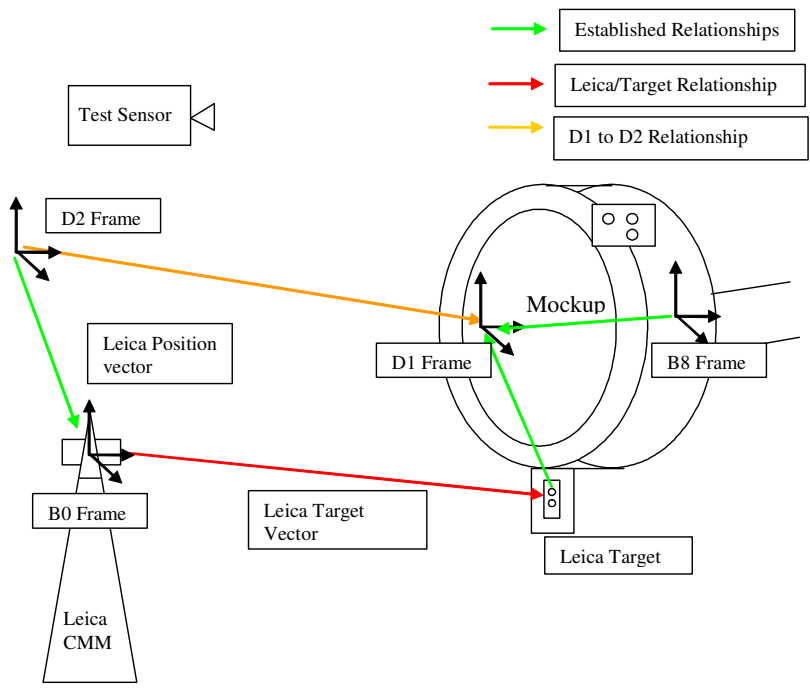

Figure 13. DOTS Vector relationships with Leica

\subsection{TEST TRAJECTORIES}

Many factors were considered in the development of the set of test trajectories. The approach was to identify a wide range of test conditions, available facilities, sensor-specific test parameters, test requirements for math model implementation, and CEV-type test conditions, and then to create a set of trajectories that represented the best combination of tests that could be conducted within the available project resources. The full test plan includes a complete list of test cases under consideration as well as a prioritization.

The test plan will be performed with the following limitations:

1. The test plan is not designed to provide the full scope of tests required for verification testing because the requirements have not been derived against which verification testing would be performed. Additionally, the pathfinder sensor hardware does necessarily meet rigorous hardware and software standards.

2. The test plan does not cover qualification testing; i.e., no flight-like hardware testing in most cases.

3. Although the overall test range applicable for these tests is from 0 to 2000 meters, the testing focus is on close range (inside 40 meters) due to facility availability and resource limitations.

4. The test plan does not include specific characterization tests for evaluating radio frequency (RF) sensors. Those tests could be added later if the CEV contractorproposed sensor suite includes RF sensors for relative navigation.

There are four types of trajectories defined for this test program. The first is a set of trajectories for sensor characterization. These trajectories cover a wide range of radial distances and velocities, azimuth and elevation angles and angular rates, and vehicle angular rates. The second type is based on reference trajectories for the CEV provided by the CEV Flight Dynamics Orbit GN\&C team. These trajectories cover approach and departure scenarios under varying dynamic conditions. The third type is a set of test cases designed to evaluate the effects of lighting conditions on the pathfinder sensors, two of which are particularly sensitive to lighting (ACVS and NFIR). The fourth type is a set of failure or extreme conditions, and includes trajectories to evaluate sensor performance in the presence of jet failure dynamic conditions, during close-in acquisition, with blockage, and with interference; e.g., multiple targets, light reflections, reflective materials.

Due to the $15 \mathrm{~m}$ limit of the Leica truth sensor, all trajectories needed to be broken into segments of $15 \mathrm{~m}$ or shorter. The segments were designed to have some overlap. In addition, some of the trajectories were designed to be run the full $40 \mathrm{~m}$ length of the facility without the Leica truth data, in order to have continuity during some of the runs.

The sensor characterization trajectories are a subset of a "Super Matrix" that defines a large range of conditions that are not achievable within the scope of this project. Table 7 shows the trajectory characteristics for the Super Matrix and the reduced set of sensor characterization tests. 
Table 7. Trajectory Characteristics for Sensor Characterization Tests

\begin{tabular}{|c|c|c|}
\hline & Super Matrix & Sensor Charact. \\
\hline \# Trajectories & & 55 \\
\hline \# Trajectory Files & & 55 \\
\hline Total Traj. Time (Hours) * & & 23.19 \\
\hline \multicolumn{3}{|l|}{ Max Relative Position } \\
\hline Radial Distance (m) & 2000.00 & 36.58 \\
\hline Azimuth (deg) & 45.00 & 10.00 \\
\hline Elevation (deg) & 45.00 & 10.00 \\
\hline \multicolumn{3}{|l|}{ Max Relative Attitude } \\
\hline Roll & $\begin{array}{c}-180 \text { to } 180 \\
\text { deg }\end{array}$ & -25 to +25 deg \\
\hline Pitch & -45 to $45 \mathrm{deg}$ & -25 to +25 deg \\
\hline Yaw & -45 to $45 \mathrm{deg}$ & -25 to +25 deg \\
\hline \multicolumn{3}{|l|}{ Max Relative Velocity } \\
\hline Radial (m/s) & 3.00 & 0.30 \\
\hline Azimuth (deg/s) & 5.00 & 1.00 \\
\hline Elevation (deg/s) & 5.00 & 1.00 \\
\hline \multicolumn{3}{|l|}{ Max Relative Attitude Rates } \\
\hline Roll Rate (deg/s) & 5.00 & 0.50 \\
\hline Pitch Rate (deg/s) & 5.00 & 0.50 \\
\hline Yaw Rate (deg/s) & 5.00 & 0.50 \\
\hline Lighting & Varying & Constant \\
\hline
\end{tabular}

Table 8 shows the trajectory characteristics for the CEV trajectories. There are two types of CEV trajectories: those broken into segments and those which span the full length of the FRL facility.
Table 8. Trajectory Characteristics for CEV Trajectory Tests

\begin{tabular}{|l|c|c|}
\hline & $\begin{array}{c}\text { CEV } \\
\text { Trajectories } \\
\text { (Segments) }\end{array}$ & $\begin{array}{c}\text { CEV } \\
\text { Trajectories } \\
\text { (Complete traj) }\end{array}$ \\
\hline \# Trajectories & 4 & 8 \\
\hline \# Trajectory Files & 4 & 8 \\
\hline Total Traj. Time (Hours) & 3.02 & 1.35 \\
\hline Max Relative Position & & \\
\hline Radial Distance (m) & 36.58 & 36.58 \\
\hline Azimuth (deg) & 5.00 & 5.00 \\
\hline Elevation (deg) & 5.00 & 5.00 \\
\hline Max Relative Attitude & & \\
\hline Roll & $3 \mathrm{deg}$ & $3 \mathrm{deg}$ \\
\hline Pitch & $2 \mathrm{deg}$ & $2 \mathrm{deg}$ \\
\hline Yaw & $1 \mathrm{deg}$ & $1 \mathrm{deg}$ \\
\hline Max Relative Velocity & & \\
\hline Radial (m/s) & 0.18 & 0.18 \\
\hline Azimuth (deg/s) & & \\
\hline Elevation (deg/s) & & \\
\hline Max Relative Attitude Rates & & \\
\hline Roll Rate (deg/s) & 0.10 & 0.10 \\
\hline Pitch Rate (deg/s) & 0.10 & 0.10 \\
\hline Yaw Rate (deg/s) & 0.10 & 0.10 \\
\hline & & \\
\hline Lighting & Constant & \\
\hline
\end{tabular}

Table 9 shows the trajectory characteristics for the lighting tests and the failure/acquisition/blockage tests. 
Table 9. Trajectory Characteristics for Lighting and Failure/Extreme Condition Tests

\begin{tabular}{|c|c|c|}
\hline & $\begin{array}{c}\text { FRL Lighting } \\
\text { Tests }\end{array}$ & $\begin{array}{c}\text { Failure, } \\
\text { Acquisition / } \\
\text { Blockage } \\
\text { Scenarios }\end{array}$ \\
\hline \# Trajectories & 5 & 13 \\
\hline \# Trajectory Files & 1 & 13 \\
\hline Total Traj. Time (Hours) & 1.25 & 2.65 \\
\hline \multicolumn{3}{|l|}{ Max Relative Position } \\
\hline Radial Distance $(\mathrm{m})$ & 36.58 & 36.58 \\
\hline Azimuth (deg) & 0.00 & \\
\hline Elevation (deg) & 0.00 & \\
\hline \multicolumn{3}{|l|}{ Max Relative Attitude } \\
\hline Roll & $0 \mathrm{deg}$ & \\
\hline Pitch & $0 \mathrm{deg}$ & \\
\hline Yaw & $0 \mathrm{deg}$ & \\
\hline \multicolumn{3}{|l|}{ Max Relative Velocity } \\
\hline Radial (m/s) & 0.18 & \\
\hline Azimuth (deg/s) & 0.00 & \\
\hline Elevation $(\mathrm{deg} / \mathrm{s})$ & 0.00 & \\
\hline \multicolumn{3}{|l|}{ Max Relative Attitude Rates } \\
\hline Roll Rate (deg/s) & 0.00 & 1.00 \\
\hline Pitch Rate (deg/s) & 0.00 & 1.00 \\
\hline Yaw Rate (deg/s) & 0.00 & 1.00 \\
\hline Lighting & $\begin{array}{c}\text { Dynamically } \\
\text { varying }\end{array}$ & Constant \\
\hline Other & & $\begin{array}{l}\text { Add blockage } \\
\text { and } \\
\text { interference for } \\
2 \text { of the CEV } \\
\text { trajectories }\end{array}$ \\
\hline
\end{tabular}

\subsection{TEST EXECUTION}

Schedule - when sensors were tested

Observations - number of runs per sensor, problem summary

\subsection{TEST RESULTS}

\section{AVGS Test Results}

The Advanced Video Guidance Sensor (AVGS) went through a test sequence in the Flight Robotics Laboratory (FRL) from July 19 to August 4, 2006. The test sequence was a series of pre-programmed trajectories through which a target moved relative to the sensor.

In order to determine the validity of the AVGS data, the AVGS data of each trajectory was examined by plotting $X$, $\mathrm{Y}$, and Z (as well as Roll, Pitch, and Yaw for trajectories with angular motion) and comparing with the nominal trajectory Leica plots. The AVGS has two separate targets for different ranges, a Short Range Target (SRT) and a Long Range Target (LRT.)

Although the final analysis has not been completed, the initial results show that the AVGS performed well. One plot is shown as an example in Figure 14. The trajectory is a failed jet (Fail_Jet2) - one that mimics a spacecraft thruster failing on. The plot is in the $\mathrm{z}$ direction (up and down in the facility). Notice that there are some straight diagonal lines those are a feature of Matlab and indicate where the AVGS stopped tracking one target or the other. The plots shows the SRT solution dropped out twice where the target went out of the field-of-view and the LRT solution dropped track when the range ( $x$ in this case) got too close. The initial results indicate the AVGS data matches fairly closely with the Leica data (positions within 3 to $6 \mathrm{~cm}$ and attitudes within .01 to .25 degrees).

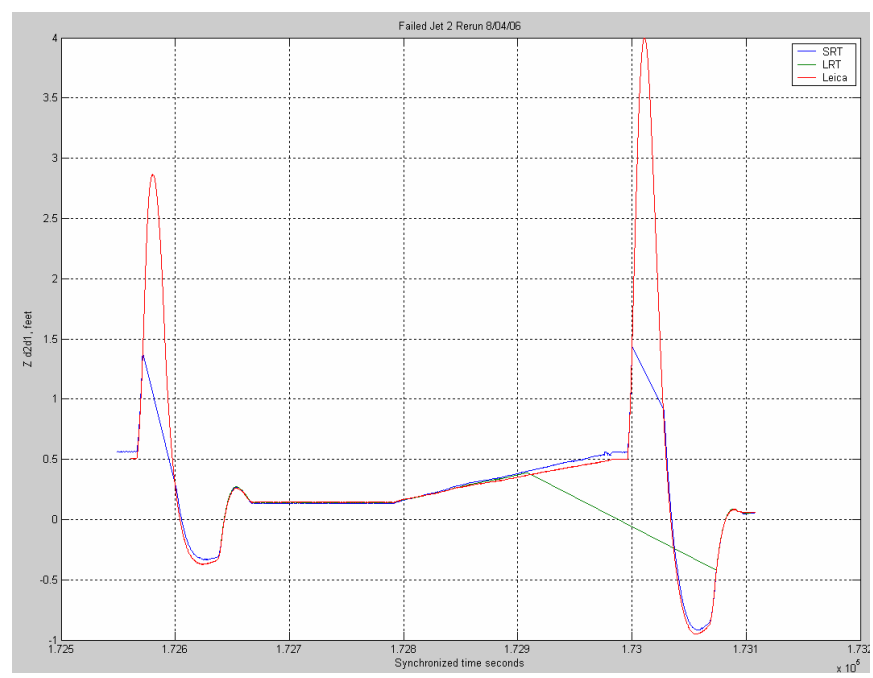

Figure 14. Plot of $Z$ (in feet) for Leica, AVGS SRT, and AVGS LRT.

\section{ACVS Test Results}

NFIR Test Results

Optech LIDAR Test Results

10.0 CONCLUSIONS 